\title{
Drag on a sphere in a spherical dispersion containing Carreau fluid
}

\author{
J.P. Hsu ${ }^{\text {a,* }}$, S.J. Yeh ${ }^{\text {b,1 }}$, S. Tseng ${ }^{c}$ \\ a Department of Chemical Engineering and Institute of Polymer Science and Engineering, National Taiwan University, Taipei, Taiwan 10617 \\ ${ }^{\mathrm{b}}$ Department of Chemical Engineering, National Taiwan University, Taipei, Taiwan 10617 \\ c Department of Mathematics, Tamkang University, Tamsui, Taipei, Taiwan 25137
}

\section{A R T I C L E I N F O}

\section{Article history:}

Received 29 November 2007

Received in revised form 17 March 2008

Accepted 24 March 2008

Available online 9 April 2008

\section{Keywords:}

Drag coefficient

Spherical dispersion

Unit cell model

Carreau fluid

\begin{abstract}
A B S T R A C T
The drag on a rigid sphere in a spherical dispersion containing Carreau fluid is investigated theoretically based on a free surface cell model for Reynolds number in the range [0.1,100], Carreau number in the range $[0,10]$, the power-law index in the range $[0.3,1]$, and the void fraction in the range $[0.271,0.999]$. The influences of the particle concentration, the nature of the Carreau fluid, and Reynolds number, on the drag coefficient are examined. We show that the drag coefficient declines with the decreasing particle concentration, and the reversal of the flow field in the rear region of a sphere is enhanced by the shearthinning nature of the fluid. An empirical relation, which correlates the drag coefficient with the void fraction ( $=1$ - particle concentration), the nature of the Carreau fluid, and Reynolds number, is proposed.
\end{abstract}

(c) 2008 Elsevier B.V. All rights reserved.

\section{Introduction}

The evaluation of the drag acting on a particle as it translates in a fluid medium is of both fundamental and practical significance. Sedimentation, which is often adopted to characterize the 2 physical properties of a particle, for example, involves this type of problem. In a dispersion of particles the drag acting on an individual particle depends on its size, shape, relative density, and the concentration of particles. In particular, the influence of the presence of neighboring particles on the drag acting on a particle is of profound nature because it involves a many-body problem. In settling, for instance, the settling speed of a dispersion of particles is slower than that of an isolated particle [1]. Unfortunately, because particles seldom present individually in practice, that influence often needs to be considered and solving the associate problem becomes non-trivial. This difficulty can be circumvented by applying a cell model, in which a dispersion is simulated by a unit cell comprising a representative particle and a concentric liquid shell. Two types of cell model are available in the literature: free surface cell model [2] and zero vorticity cell model [3]. These models are essentially the same in all aspects except that zero shear stress and zero vorticity are assumed on the cell boundary in the former and in the latter, respectively.

The cell model has been applied successfully to simulate the behaviors and to calculate the physical properties of various types of dispersed system. These include, for instance, the flow through a

\footnotetext{
* Corresponding author. Fax: +886223623040.

E-mail addresses: jphsu@ntu.edu.tw (J.P. Hsu), f92524027@ntu.edu.tw (S.J. Yeh), topology@mail.tku.edu.tw (S. Tseng).

1 Fax: +886223623040
}

porous medium [4], permeability and viscosity studies [4], the pressure drop in packed and fluidized beds [4,5], the motion and mass transfer of a bubble swarm [6], the settling velocity of a rigid spherical dispersion [7], the sedimentation of rigid spheres [8], the mass transfer of spherical particles in assemblages [9-11], the sedimentation of a dispersion of spherical composite particles and the fluid flow through a bed of spherical composite particles [12], and the drag on a non-uniformly structured floc in a floc dispersion [13].

If the concentration of a dispersion is appreciable, it is usually of non-Newtonian nature [14-17]. In this case, because the viscosity of the dispersion is position dependent, the evaluation of the drag acting on a particle is much more complicated than that in the corresponding Newtonian case. Several attempts have been made to solve this type of problem. Kawase and Ulbrecht [18], for example, applied a free surface cell model and a boundary layer theory to analyze the behavior of an assemblage of rigid spheres in a power-law fluids at high Reynolds numbers. Adopting free surface cell model [2] and variational principles, Chhabra and Raman [19] were able to obtain bounds on the drag for the creeping flow of a Carreau fluid past an assemblage of rigid spheres. Staish and Zhu [20] and Jaiswal et al. [21,22] solved numerically the problem of an unbounded slow flow of nonNewtonian fluids (power-law or Carreau model) through an assemblage of rigid spheres. Using both a free surface cell model and a zero vorticity cell model, Ferreira et al. $[23,24]$ considered the steady flow of an incompressible power-law fluid across an assemblage of rigid cylinders. Note that both the free surface and zero vorticity cell models are idealizations, and there is no justification for either of these boundary conditions. In a discussion of cell models, Zholkovskiy et al. [25] pointed out that if the particle volume fraction is sufficiently low, the zero vorticity cell model performs better than the free surface cell 


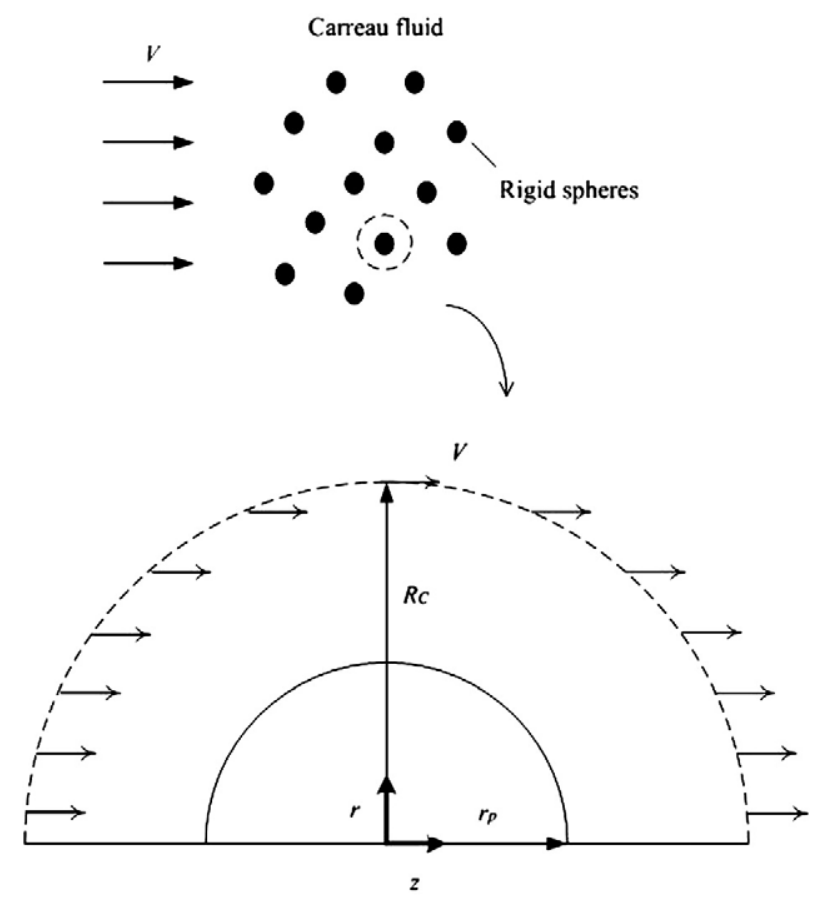

Fig. 1. Steady translation of rigid spherical particles of radius $r_{\mathrm{p}}$ in a Carreau fluid. For convenience, the spheres are fixed in the space and the velocity of the approaching liquid is $V$. The system is simulated by a unit cell model where it is mimicked by a cell comprising a representative sphere and a concentric spherical liquid shell of radius $R_{\mathrm{c}}$. The cylindrical coordinates are adopted with its origin located at the center of the cell, and $r$ and $z$ are the radial and the axial coordinates, respectively.

model does. However, if the volume fraction approaches the value attributed to the close packing, the free surface cell model becomes the better choice among other models, including the zero vorticity cell model.

In this study, the drag on a rigid sphere in a spherical dispersion containing Carreau fluid is estimated based on a free surface cell model for the case of small to medium large Reynolds number. The influences of the concentration of particles, the Reynolds number, and the properties of a Carreau fluid on the drag coefficient are investigated. Empirical relationships that correlate the drag coefficient with the key parameters of the system under consideration are proposed.

\section{Theory}

Let us consider the steady translation of rigid spheres of radius $r_{\mathrm{p}}$ in a liquid. For convenience, the spheres are fixed in the space and the velocity of the approaching liquid is $V$. Referring to Fig. 1, a unit cell model is adopted where the spherical dispersion is simulated by a cell comprising a representative sphere and a concentric spherical liquid shell of radius $R_{\mathrm{c}}$. The cylindrical coordinates are adopted with its origin located at the center of the cell, and $r$ and $z$ are the radial and the axial coordinates, respectively. The concentration of the spherical dispersion can be estimated by $(1-\varepsilon)$, where $\varepsilon$ is the void fraction defined by

$\varepsilon=1-\left(\frac{r_{\mathrm{p}}}{R_{\mathrm{c}}}\right)^{3}$

Suppose that the liquid phase is incompressible. Then the flow field can be described by

$\rho \mathbf{u} \cdot \nabla \mathbf{u}=-\nabla P+\nabla \cdot \boldsymbol{\tau}$

$\nabla \cdot \mathbf{u}=0$ where $\rho$ is the density of the liquid phase, $P$ is the pressure, $\nabla$ is the gradient operator, $\tau$ is the stress tensor, and $\mathbf{u}$ is the liquid velocity. For a generalized Newtonian fluid [26-29]

$\tau=-\eta(\dot{\gamma}) \dot{\gamma}$

where $\dot{\gamma}=\left(\nabla \mathbf{u}+(\nabla \mathbf{u})^{T}\right)$ is the rate-of-strain tensor, $\dot{\gamma}$ is its magnitude, $\eta$ is the apparent viscosity, and the superscript $T$ denotes matrix transpose. Suppose that the liquid phase is a Carreau fluid, the viscosity of which can be described by [26-29]

$\eta(\dot{\gamma})=\eta_{0}\left[1+(\lambda \dot{\gamma})^{2}\right]^{(n-1) / 2}$

where $\eta_{0}$ is the viscosity corresponding to the minimum shear rate, $\lambda$ is the relaxation time constant, and $n$ is the power-law index. Note that if $n=1$ and/or $\lambda=0$, the liquid becomes Newtonian.

The following boundary conditions are assumed for the flow field:

$u_{z}=V$ at $r=R_{\mathrm{c}}$

$\tau_{r \theta}=0$ at $r=R_{\mathrm{c}}$

$u_{z}=0$ on the sphere surface

where $u_{z}$ is the fluid velocity in the $z$-direction.

\section{Results and discussion}

FIDAP7.6, a commercial software based on a finite element method, is adopted to solve the governing equations and the associated boundary conditions. Throughout the computation, double precision is used and grid independence is checked. In our case, using roughly 7000 elements in the liquid domain is sufficient. Fig. 2 illustrates the typical mesh used. The applicability of the software adopted is justified by applying it to the case of a Newtonian fluid and comparing the result obtained with the available result in the literature. The drag acting on an isolated rigid sphere in a fluid, $F_{\mathrm{D}}$, can be expressed as $[26,30-32]$

$F_{\mathrm{D}}=\left(\frac{1}{2} \rho V^{2}\right)\left(\pi r_{\mathrm{p}}^{2}\right) C_{\mathrm{D}}$

where $C_{\mathrm{D}}$ is the drag coefficient. Fig. 3 shows the variation of $F_{\mathrm{D}}$ as a function of $\varepsilon$ for the case of a Newtonian fluid under a creeping flow condition. Both the analytical result of Happel and Brenner [4] and the numerical result based on the present approach are presented. As seen in Fig. 3, the performance of the software adopted is satisfactory.

For illustration, we assume that $\eta_{0}=3 \mathrm{~g} / \mathrm{cm} \mathrm{s}$, and therefore the parameters key to the behavior of a sphere are the Reynolds number, the concentration of spheres, and the properties of the Carreau fluid. The influences of these parameters on the flow field and the drag coefficient are investigated through numerical simulation. For convenience, we define the Carreau number $\mathrm{Cu}$ and the Reynolds number Re as [26-29]

$C u=\lambda V / r_{\mathrm{p}}$

$R e=2 \rho r_{\mathrm{p}} V / \eta_{0}$.

The simulated flow fields near a sphere at various combinations of the void fraction $\varepsilon$, the Carreau number $C u$, and the Reynolds number

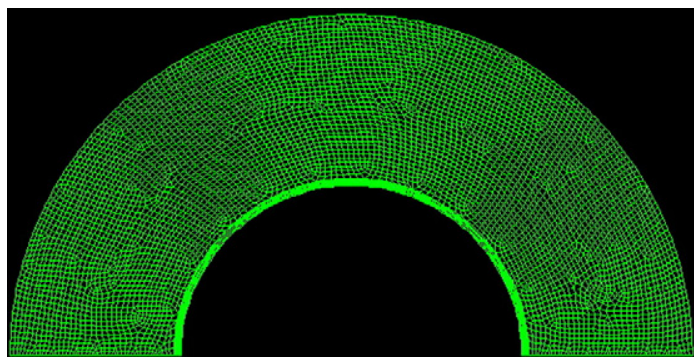

Fig. 2. Typical mesh used in the numerical procedure. 


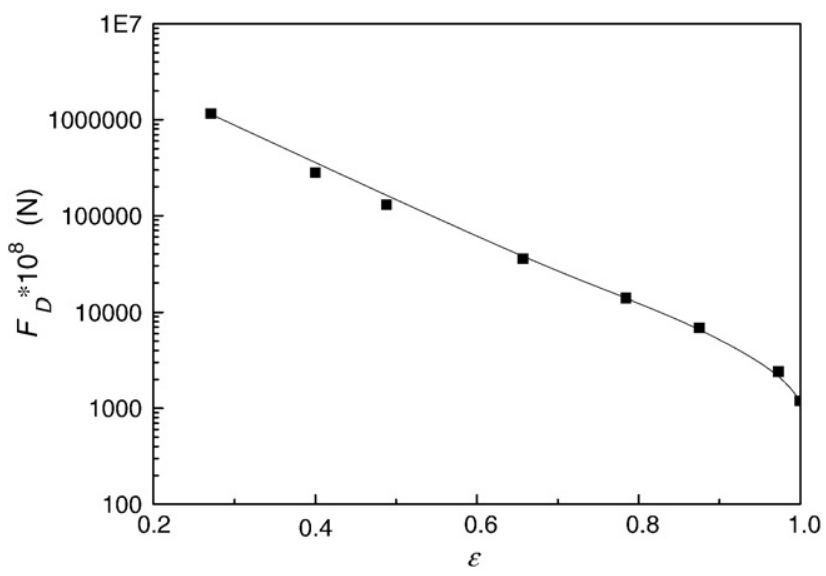

Fig. 3. Variation of the drag on a rigid sphere in a spherical dispersion of Newtonian fluid as a function of $\varepsilon$ for various values of $\left(r_{\mathrm{p}} / R_{\mathrm{c}}\right)$ at $R e=0.01$. Solid curve, result of Happel and Brenner [4], discrete symbols, present numerical results.

Re are illustrated in Figs. 4-6. These figures suggest that if $R e$ is sufficiently small, the flow field in the front region of a sphere is essentially symmetric to that in its rear region, regardless of the

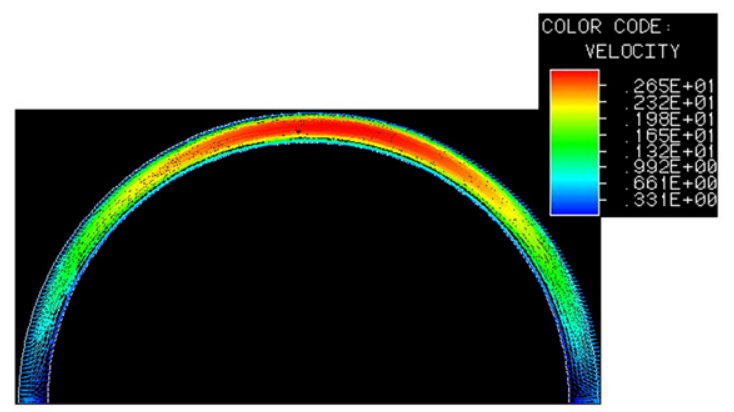

(a)

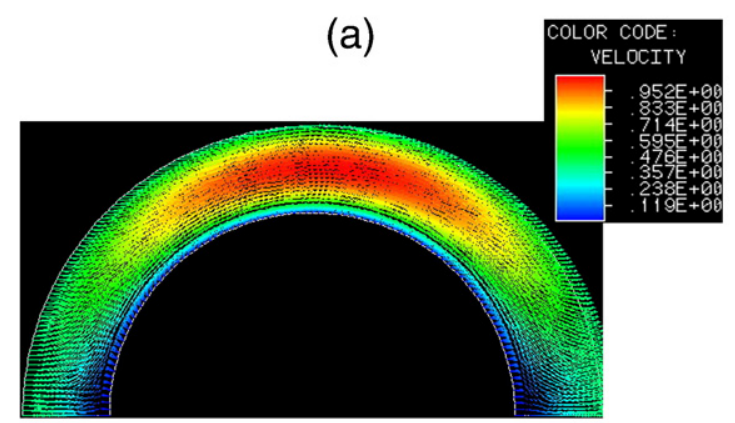

(b)

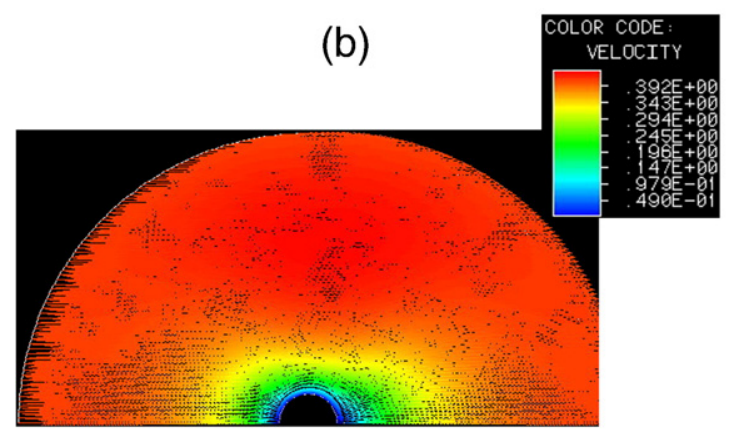

(c) levels of $\varepsilon$ and $\mathrm{Cu}$. However, as Re becomes large, they are no longer symmetric to each other. In this case, a reverse flow is present and vortexes can be observed in the rear region of the sphere. The presence of the neighboring spheres has the effect of confining this reverse flow so that the reverse of the flow field in the rear region of a sphere occurs only if $\varepsilon$ is sufficiently large. On the other hand, the shear-thinning nature of the fluid has the effect of enhancing the reverse flow. Note that if the shear-thinning nature of the fluid is significant, such as in Fig. 6(d), although $\varepsilon$ is not large enough to observe the reverse flow in the rear region of the sphere, the flow field there becomes unstable, and local turbulent flow is present.

Fig. 7 shows the influence of particle concentration on the drag coefficient $C_{D}$ for various combinations of $\mathrm{Cu}$ and $R e$. This figure reveals that for the range of $R e$ considered, regardless of the magnitude of $R e$, the qualitative behaviors of $C_{D}$ are roughly the same. In general, for a fixed $C u, C_{D}$ decreases with the increasing in $\varepsilon$ (decreasing in the particle concentration), and the smaller the $\varepsilon$ (higher the particle concentration) the more significant is its influence on $C_{D}$. For a fixed $\varepsilon, C_{D}$ declines with the increase in $\mathrm{Cu}$, which is expected because the larger the $\mathrm{Cu}$ the more important the effect of shear thinning is. Also, the larger the $\varepsilon$ (lower the particle concentration) the more significant the
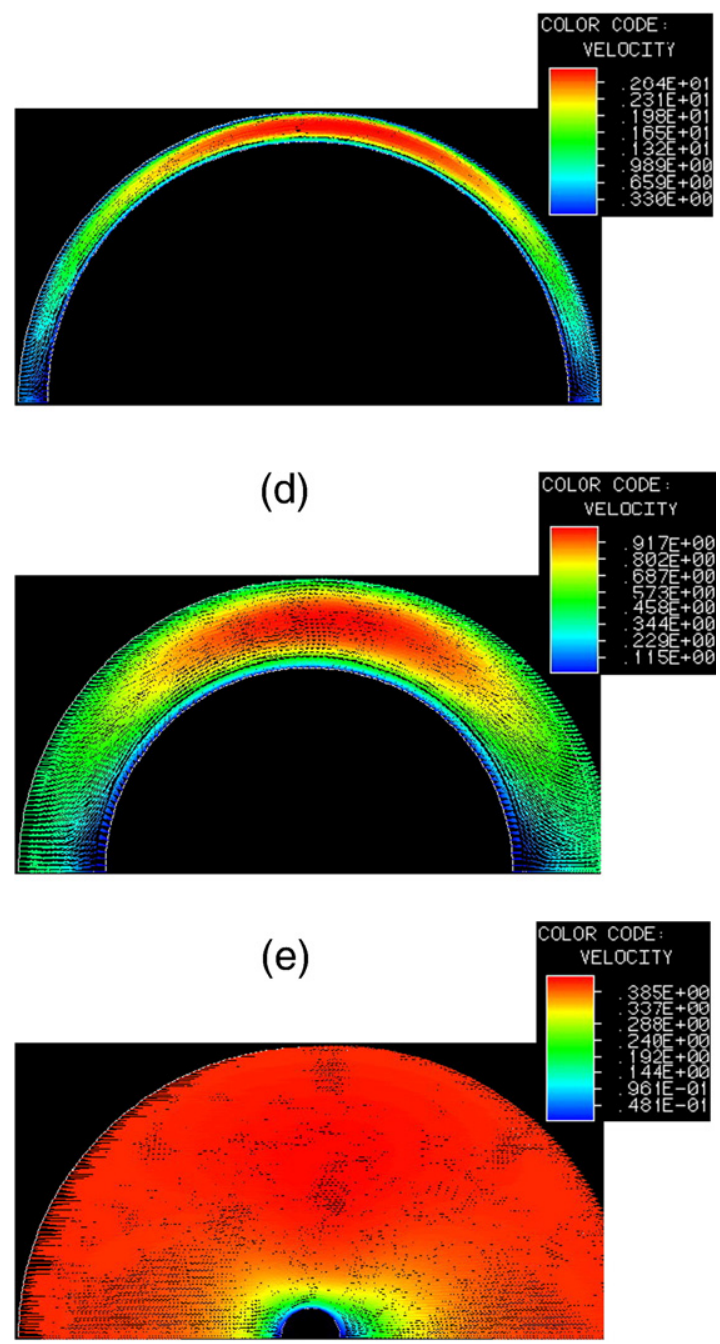

(f)

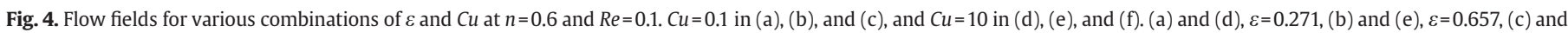
(f), $\varepsilon=0.999$. 

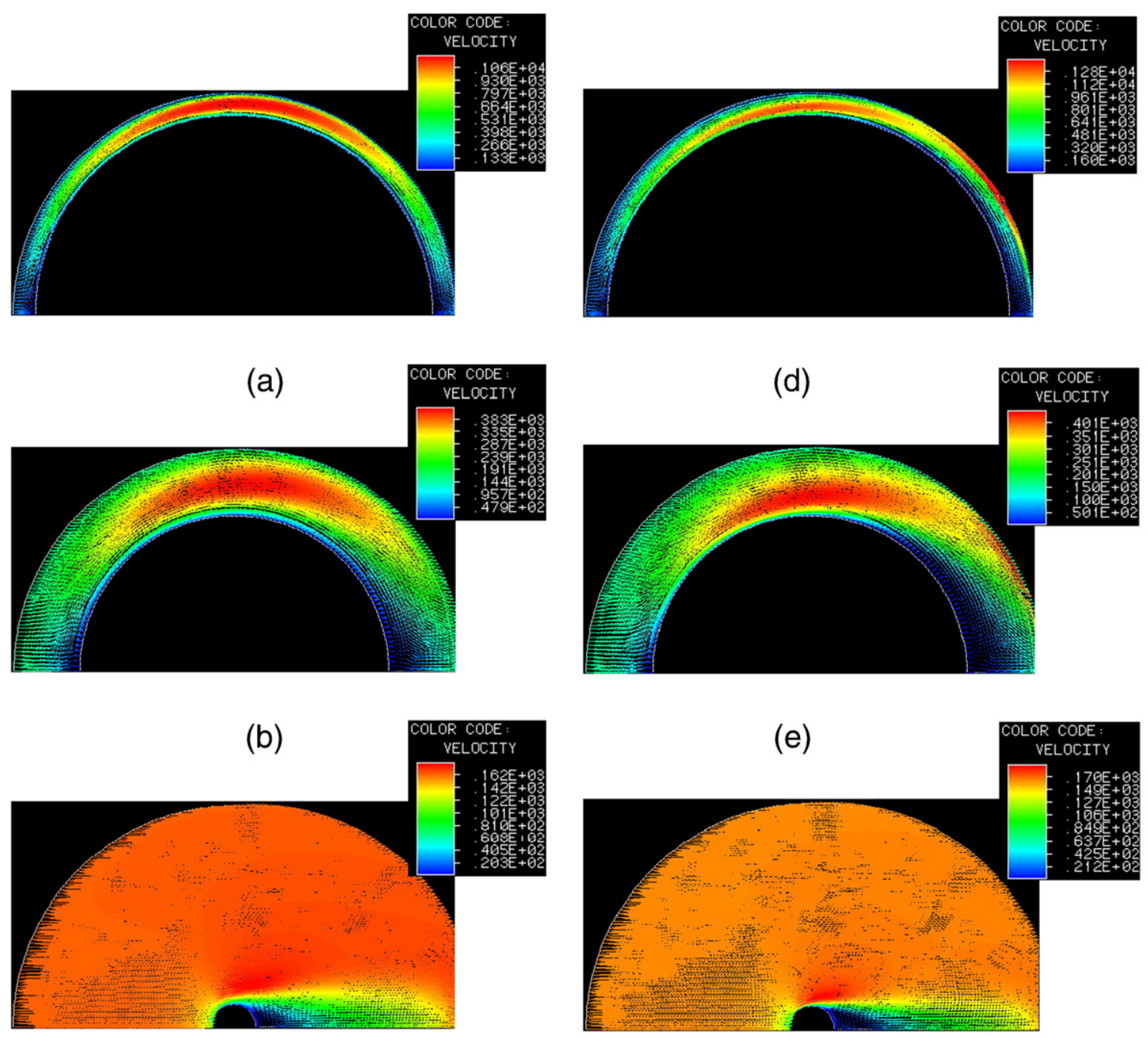

(c)

(f)

Fig. 5. Flow fields for various combinations of $\varepsilon$ and $C u$ at $n=0.6$ and $R e=40$. $C u=0.1$ in (a), (b), and (c), and $C u=10$ in (d), (e), and (f). (a) and (d), $\varepsilon=0.271$, (b) and (e), $\varepsilon=0.657$, (c) and (f), $\varepsilon=0.999$.

influence of $\mathrm{Cu}$ is, and this phenomenon is pronounced if $n$ becomes smaller.

The influence of the particle concentration on the drag coefficient, measured by the ratio $R=\left[C_{\mathrm{D}}(\varepsilon=0.271) / C_{\mathrm{D}}(\varepsilon=0.999)\right]$, at various combinations of $n, R e$, and $C u$ is summarized in Table 1. As seen from this table, the influence of the particle concentration is more important at a smaller $\mathrm{Cu}$, a smaller $\mathrm{Re}$, and a larger $n$, implying that the shear-thinning nature of a fluid has the effect of reducing the influence of particle concentration. It is interesting to observe that at $n=0.6$ and $C u=10, R$ has a local minimum as $R e$ varies. A local minimum in $R$ is also observed as $C u$ varies at $n=0.6$ and $R e=100$, and as $R e$ varies at both $C u=0.5$ and $C u=1$ for $n=0.5$, 0.4 , and 0.3 . In general, the more important the shear-thinning nature of a fluid the easier for $R$ to have a local minimum. The presence of the local minimum arises from the specific behavior of the flow field in the rear region of a sphere such as that shown in Fig. 6(d).

From application point of view, it is highly desirable to correlate $C_{D}$ with the parameters key to the system under consideration. To this end, a regression analysis is conducted based on the simulation data gathered in our study. For $0.5<n \leqq 0.8,0.1 \leqq C u \leqq 10, \varepsilon \leqq 0.7$, and $R e \leqq 100$, we arrive at

$$
\begin{aligned}
& C_{\mathrm{D}}=\frac{A}{C u^{(B-C \times R e)} \operatorname{Re}^{D} \varepsilon^{[E+F \exp (-R e / G)]}} \\
& A=11.7+170 n \\
& B=0.9455-0.935 n \\
& C=0.00617-0.007 n \\
& D=0.844+0.12 n \\
& E=2.72384-2.06055 \times 10^{-12} \exp \left(\frac{n}{0.02835}\right) \\
& F=2.33773 \times 10^{-7} \exp \left(\frac{n}{0.04779}\right) \\
& G=3.19373 \times 10^{-5} \exp \left(\frac{n}{0.0456}\right) .
\end{aligned}
$$



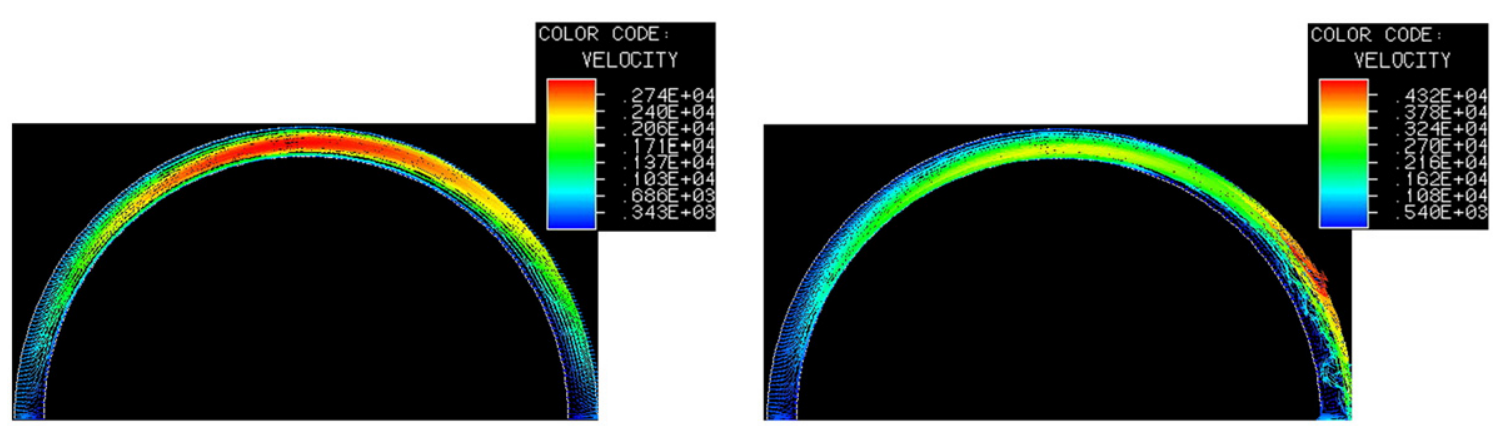

(a)

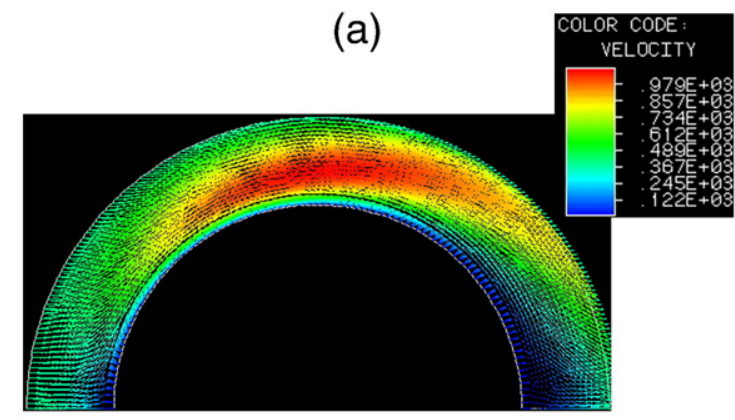

(b)

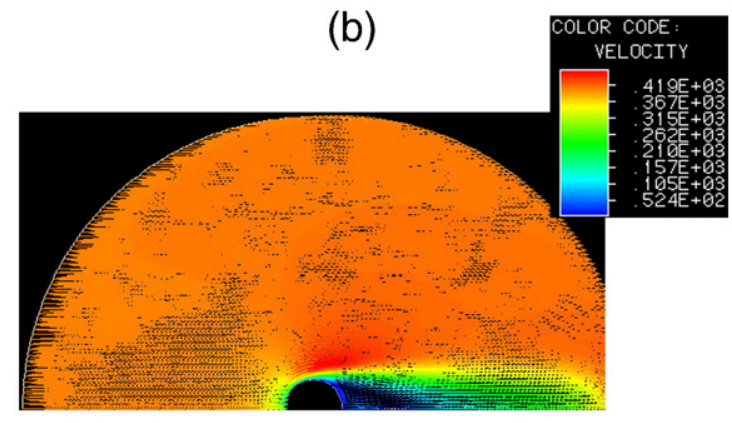

(c)

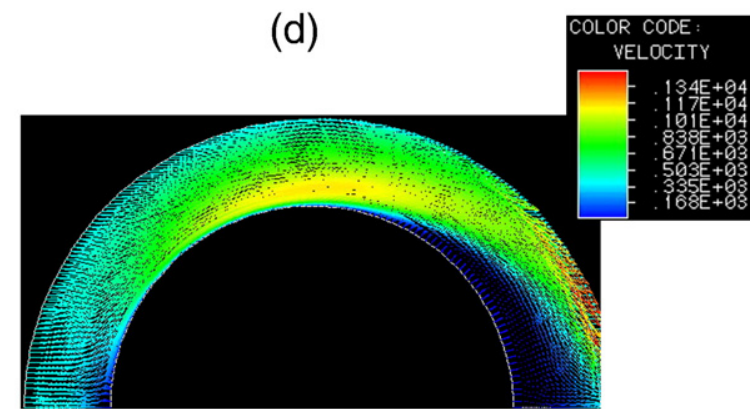

(e)

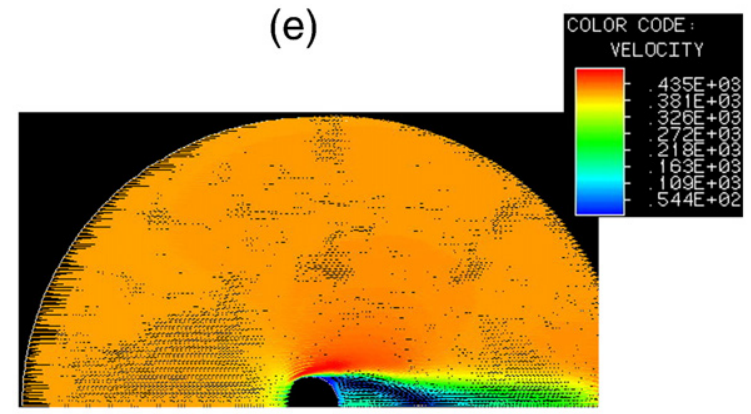

(f)

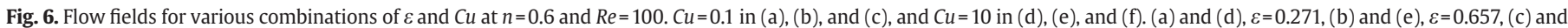
(f), $\varepsilon=0.999$.

For $0.3 \leqq n \leqq 0.5,0.1 \leqq C u \leqq 1, \varepsilon \leqq 0.7$, and $R e \leqq 40$, we obtain

$C_{\mathrm{D}}=\frac{A^{\prime}}{C u^{\left(B^{\prime}-C^{\prime} \times R e\right)} R e^{D \prime} \varepsilon^{E^{\prime}}}$

$A^{\prime}=43.333+110 n$

$B^{\prime}=0.90533-0.86 n$

$C^{\prime}=0.00547-0.007 n$

$D^{\prime}=0.89133+0.06 n$

$E^{\prime}=1.47333+2.05 n$

For $0.8<n \leqq 0.9,0.1 \leqq C u \leqq 10, \varepsilon \leqq 0.7$, and $R e \leqq 100, C_{D}$ can be described by

$C_{\mathrm{D}}=\frac{169}{C u^{(0.099-0.0002 R e)} R e^{0.954} \varepsilon^{(3.656-0.0031 R e)}}$.

Fig. 8 summarizes the variation of the $C_{D}$ based on the correlation relationships, Eqs. (12)-(14), as a function of the $C_{D}$ calculated in this study. In general, the performance of the correlation relationships obtained is satisfactory. Although the maximum deviation is on the order of $30 \%$, only few points deviate appreciably. In general, the larger the value of $n$ the better the performance of Eqs. (12)-(14).

The performance of the present model is further examined by comparing the results calculated with those predicted by other theoretical models $[20,21,33,34]$, where a power law, shear-thinning fluid in the creeping flow region was considered with

$\eta(\dot{\gamma})=K|\dot{\gamma}|^{m-1}$.

The values of the parameters assumed are $K=20 \mathrm{~g} /\left(\mathrm{cm}^{2} \mathrm{~s}\right)$ and $m=0.6$, which correspond approximately to $\eta_{0}=100$ poise, $\lambda=55.90 \mathrm{~s}$, and $n=0.6$ in the present Carreau model. For $\operatorname{Re}_{n}=\rho V^{2-m}\left(2 r_{\mathrm{p}}\right)^{m} / K \ll 1$, a drag correction factor $X$ is defined as [35]

$X(\varepsilon, n)=\frac{C_{\mathrm{D}} R e_{n}}{24}$.

Fig. 9 illustrates the variation of $X$ as a function of $\varepsilon$ based on the present model and that based on the other theoretical models. As seen, the general trend of $X$ is consistent with that predicted by other 

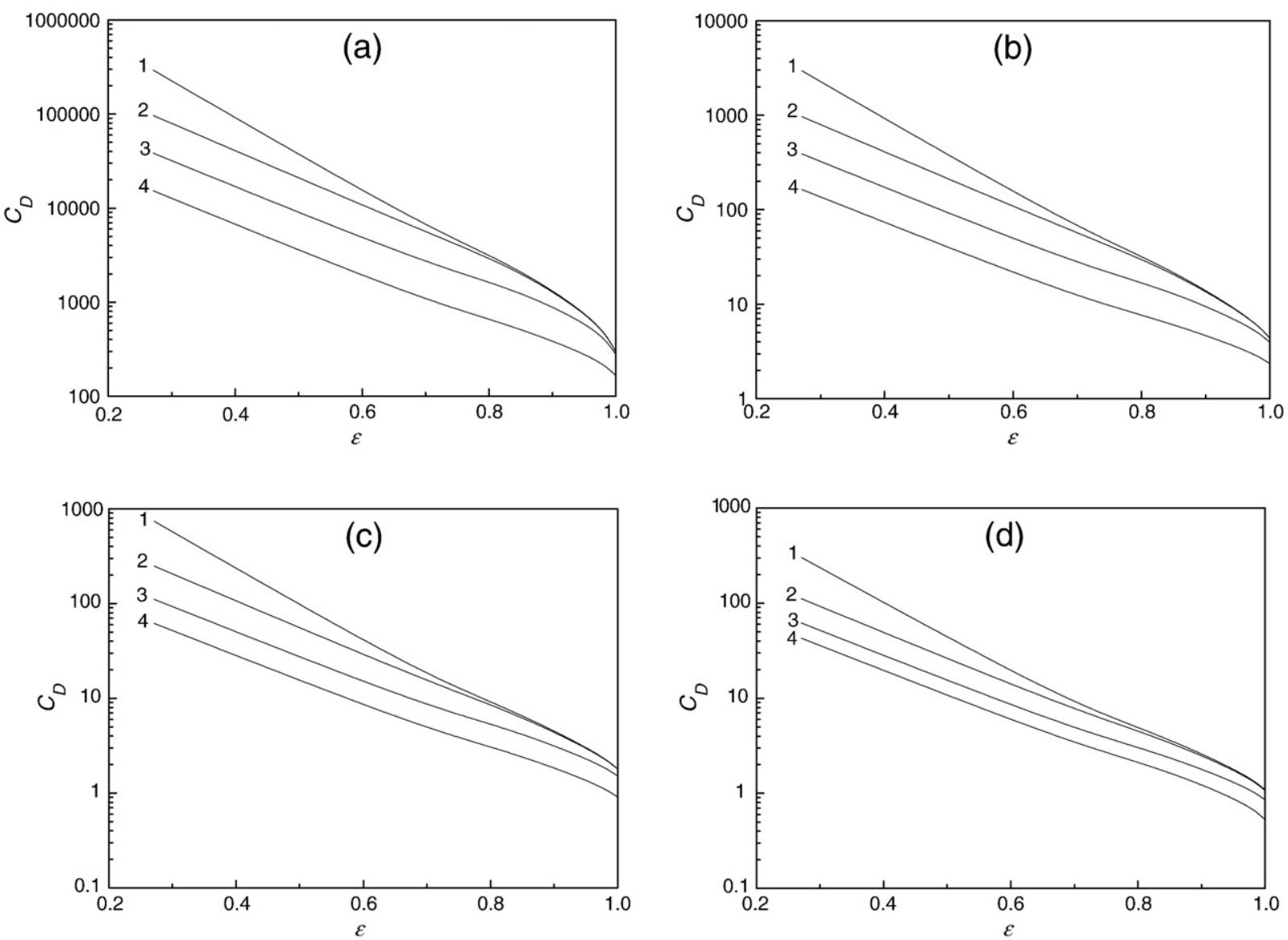

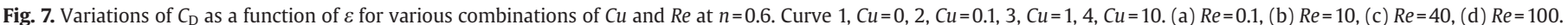

models. Fig. 9 reveals that the larger the $\varepsilon$ the closer the $X$ evaluated by the present model to that of the other models. This might arise from that the higher the concentration of particles ( $\varepsilon$ is smaller) the greater the difference between the nature of the present Carreau fluid and that of the corresponding power-law fluid. This can be justified by the experimental observation of Machač and Lecjaks [36], where the terminal velocity of a sphere in a rectangular duct filled with a nonNewtonian fluid is studied. Kerafloc, the polymer solution used in their Fig. 5, can be described by a Carreau fluid. They found that if the boundary effect is insignificant, the wall factor for the case of a powerlaw fluid [37] is close to that of a Carreau fluid. On the other hand, if the boundary effect is significant, then the difference between the two is appreciable. These imply that the smaller the $\varepsilon$ the greater the difference between the $X$ calculated by the present model and that predicted by the other power-law based models, as is seen in Fig. 9.

\section{Conclusion}

The drag on a rigid sphere in a spherical dispersion containing Carreau fluid is estimated based on a free surface cell model. Numerical simulations are conducted for Reynolds number in the range $[0.1,100]$, Carreau number in the range $[0,10]$, the power-law index in the range [0.3,1], and the void fraction in the range [0.271,0.999]. We show that if Reynolds number is sufficiently small, the flow field in the front region of a sphere is essentially symmetric to that in its rear region; if it is large, a reverse flow is present in the rear region of the sphere and the flow fields are no longer symmetric. The presence of the neighboring spheres has the effect of confining this reverse flow, but the shear-thinning nature a fluid has the effect of enhancing that flow. The drag coefficient declines with the decreasing in the particle concentration and the higher the particle concentration the more significant is its influence on the drag coefficient. An increase in the Carreau number has the effect of reducing the drag coefficient, and the lower the particle concentration and/or the smaller the power-law index the more significant that effect is. The degree of influence of the particle concentration on the drag coefficient may have a local minimum as Carreau number or Reynolds number varies; the more important the shear-thinning nature of a fluid the easier for that influence to have a local minimum. Empirical relationships are developed, which correlate the drag coefficient with Reynolds number, Carreau number, the power-law index, and the particle concentration.

\section{Acknowledgment}

This work is supported by the National Science Council of the Republic of China.

\section{References}

[1] B.H. Kaye, R.P. Boardman, Proceeding Symposium Interaction between Fluids and Particles, British Institute of Chemical Engineers, London, 1962.

[2] J. Happel, Viscous flow in multiparticle systems-slow motion of fluids relative to beds of spherical particles, AIChE Journal 4 (1958) 197-201.

[3] S. Kuwabara, The forces experienced by randomly distributed parallel circular cylinders or spheres in a viscous flow at small Reynolds numbers, Journal of the Physical Society of Japan 14 (1959) 527-532.

[4] J. Happel, H. Brenner, Low Reynolds Number Hydrodynamics, Academic Press, New York, 1983.

[5] M.M. Elkaissy, G.M. Homsy, Theoretical study of pressure-drop and transport in packed-beds at intermediate Reynolds-numbers, Industrial \& Engineering Chemistry Fundamentals 12 (1973) 82-90.

[6] B.P. LeClair, A.E. Hamielec, Viscous flow through particle assemblages at intermediate Reynolds-numbers - cell model for transport in bubble swarms, Canadian Journal of Chemical Engineering 49 (1971) 713-720.

[7] G.K. Batchelor, Sedimentation in a dilute dispersion of spheres, Journal of Fluid Mechanics 52 (1972) 245-268. 
Table 1

Influence of the particle concentration on the drag coefficient, measured by the ratio $R=$ $\left[C_{\mathrm{D}}(\varepsilon=0.271) / C_{\mathrm{D}}(\varepsilon=0.999)\right]$, at various combinations of $n, R e$, and $C u$

\begin{tabular}{|c|c|c|c|}
\hline$N$ & $R e$ & $\mathrm{Cu}$ & $\mathrm{R}$ \\
\hline \multirow[t]{12}{*}{0.9} & 0.1 & 0.1 & 732.30 \\
\hline & & 1 & 590.64 \\
\hline & & 10 & 530.50 \\
\hline & 10 & 0.1 & 502.72 \\
\hline & & 1 & 408.75 \\
\hline & & 10 & 365.86 \\
\hline & 40 & 0.1 & 309.78 \\
\hline & & 1 & 256.27 \\
\hline & & 10 & 229.74 \\
\hline & 100 & 0.1 & 212.00 \\
\hline & & 1 & 180.63 \\
\hline & & 10 & 165.16 \\
\hline \multirow[t]{12}{*}{0.8} & 0.1 & 0.1 & 554.26 \\
\hline & & 1 & 361.14 \\
\hline & & 10 & 292.85 \\
\hline & 10 & 0.1 & 380.71 \\
\hline & & 1 & 252.29 \\
\hline & & 10 & 203.96 \\
\hline & 40 & 0.1 & 235.74 \\
\hline & & 1 & 163.23 \\
\hline & & 10 & 135.45 \\
\hline & 100 & 0.1 & 164.67 \\
\hline & & 1 & 124.15 \\
\hline & & 10 & 112.12 \\
\hline \multirow[t]{12}{*}{0.7} & 0.1 & 0.1 & 419.00 \\
\hline & & 1 & 221.10 \\
\hline & & 10 & 163.14 \\
\hline & 10 & 0.1 & 288.00 \\
\hline & & 1 & 156.19 \\
\hline & & 10 & 115.91 \\
\hline & 40 & 0.1 & 179.66 \\
\hline & & 1 & 106.62 \\
\hline & & 10 & 88.45 \\
\hline & 100 & 0.1 & 129.12 \\
\hline & & 1 & 90.96 \\
\hline & & 10 & 88.39 \\
\hline \multirow[t]{12}{*}{0.6} & 0.1 & 0.1 & 316.20 \\
\hline & & 1 & 134.89 \\
\hline & & 10 & 91.10 \\
\hline & 10 & 0.1 & 217.57 \\
\hline & & 1 & 97.27 \\
\hline & & 10 & 68.93 \\
\hline & 40 & 0.1 & 137.24 \\
\hline & & 1 & 72.75 \\
\hline & & 10 & 67.40 \\
\hline & 100 & 0.1 & 102.00 \\
\hline & & 1 & 72.00 \\
\hline & & 10 & 80.00 \\
\hline \multirow[t]{12}{*}{0.5} & 0.1 & 0.1 & 238.00 \\
\hline & & 0.5 & 109.71 \\
\hline & & 1 & 82.57 \\
\hline & 10 & 0.1 & 164.04 \\
\hline & & 0.5 & 78.22 \\
\hline & & 1 & 61.26 \\
\hline & 40 & 0.1 & 105.20 \\
\hline & & 0.5 & 59.13 \\
\hline & & 1 & 53.08 \\
\hline & 100 & 0.1 & 82.17 \\
\hline & & 0.5 & 60.26 \\
\hline & & 1 & 59.82 \\
\hline \multirow[t]{12}{*}{0.4} & 0.1 & 0.1 & 178.51 \\
\hline & & 0.5 & 70.66 \\
\hline & & 1 & 50.61 \\
\hline & 10 & 0.1 & 123.31 \\
\hline & & 0.5 & 51.62 \\
\hline & & 1 & 39.46 \\
\hline & 40 & 0.1 & 80.84 \\
\hline & & 0.5 & 44.60 \\
\hline & & 1 & 41.40 \\
\hline & 100 & 0.1 & 67.23 \\
\hline & & 0.5 & 49.82 \\
\hline & & 1 & 49.39 \\
\hline \multirow[t]{3}{*}{0.3} & 0.1 & 0.1 & 133.06 \\
\hline & & 0.5 & 45.30 \\
\hline & & 1 & 30.53 \\
\hline
\end{tabular}

Table 1 (continued)

\begin{tabular}{llll}
\hline$N$ & $\mathrm{Re}$ & $\mathrm{Cu}$ & $\mathrm{R}$ \\
\hline 0.3 & 10 & 0.1 & 92.26 \\
& & 0.5 & 34.30 \\
& 40 & 1 & 26.39 \\
& & 0.1 & 62.19 \\
& 0.5 & 34.50 \\
& 1 & 33.09 \\
\hline
\end{tabular}

[8] A.K. Jaiswal, T. Sundararajan, R.P. Chhabra, Hydrodynamics of Newtonian fluidflow through assemblages of rigid spherical-particles in intermediate Reynoldsnumber regime, International Journal of Engineering Science 29 (1991) 693-708.

[9] Z.S. Mao, Numerical simulation of viscous flow through spherical particle assemblage with the modified cell model, Chinese Journal of Chemical Engineering 10 (2002) 149-162.

[10] Z.S. Mao, J.Y. Chen, Numerical approach to the motion and external mass transfer of a drop swarm by the cell model, Proc. ISEC 2002, Capetown, South Africa, 2002.

[11] Z.S. Mao, Y. Wang, Numerical simulation of mass transfer of a spherical particle assemblage with the cell model, Powder Technology 134 (2003) 145-155.

[12] H.J. Keh, Y.C. Chang, Creeping motion of an assemblage of composite spheres relative to a fluid, Colloid and Polymer Science 283 (2005) 627-635.

[13] J.P. Hsu, M.C. Li, A.C. Chang, Drag of a dispersion of nonhomogeneously structured flocs in a flow field, Journal of Colloid and Interface Science 284 (2005) 332-338.

[14] R.P. Chhabra, C. Tiu, P.H.T. Uhlherr, Creeping motion of spheres through Ellis model fluids, Rheologica Acta 20 (1981) 346-351.

[15] A. Tripathi, R.P. Chhabra, Slow power law fluid-flow relative to an array of infinite cylinders, Industrial \& Engineering Chemistry Research 31 (1992) 2754-2759.

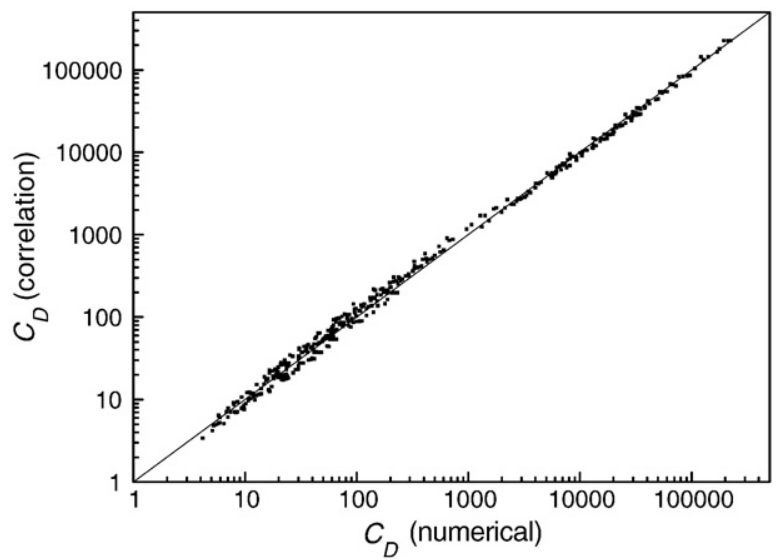

Fig. 8. Variation of the $C_{D}$ based on the correlation relationships, Eqs. (12)-(14), as a function of the $C_{\mathrm{D}}$ calculated in this study.

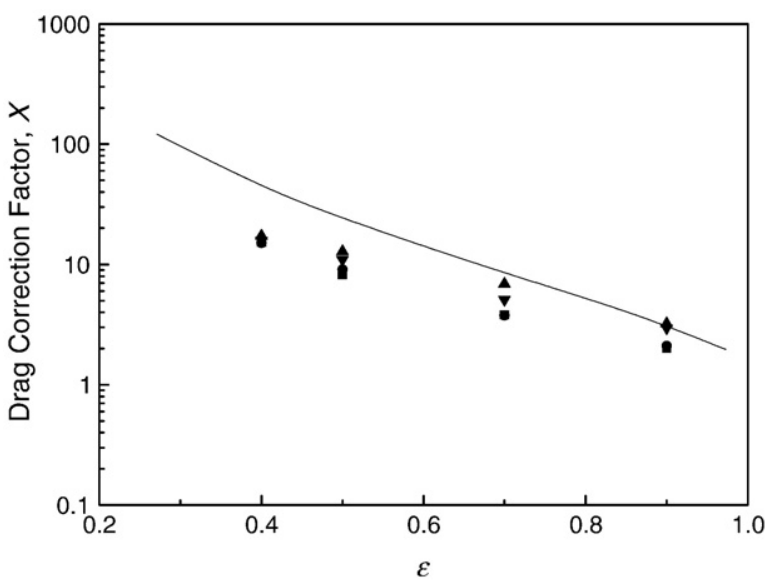

Fig. 9. Variation of the drag correction factor $(X)$ of non-Newtonian fluid as a function of $\varepsilon$ for the case where $R e_{n}=0.01, \eta_{0}=100$ poise, $\lambda=55.90 \mathrm{~s}$, and $n=0.6$. Solid curve, result predicted by the present analysis; (ロ) Jaiswal et al. [21]; (•) Satish and Zhu [20]; ( Kawase and Ulbrecht [33]; ( $\mathbf{\nabla})$ Mohan and Raghuraman [34]. 
[16] M.V. Bruschke, S.G. Advani, Flow of generalized Newtonian fluids across a periodic array of cylinders, Journal of Rheology 37 (1993) 479-498.

[17] Y.J. Liu, D.D. Joseph, Sedimentation of particles in polymer-solutions, Journal of Fluid Mechanics 255 (1993) 565-595.

[18] Y. Kawase, J.J. Ulbrecht, Motion of and mass-transfer from an assemblage of solid spheres moving in a non-Newtonian fluid at high Reynolds-numbers, Chemical Engineering Communications 8 (1981) 233-249.

[19] R.P. Chhabra, J.R. Raman, Slow non-Newtonian flow past an assemblage of rigid spheres, Chemical Engineering Communications 27 (1984) 23-46.

[20] M.G. Satish, J. Zhu, Flow resistance and mass-transfer in slow non-Newtonian flow through multiparticle systems, Journal of Applied Mechanics 59 (1992) 431-437.

[21] A.K. Jaiswal, T. Sundararajan, R.P. Chhabra, Hydrodynamics of creeping flow of power law fluids through particle assemblages, International Journal of Engineering Science 31 (1993) 293-306.

[22] A.K. Jaiswal, T. Sundararajan, R.P. Chhabra, Slow non-Newtonian flow through packed beds effect of zero shear viscosity, The Canadian Journal of Chemical Engineering 71 (1993) 646-651.

[23] J.M. Ferreira, R.P. Chhabra, Analytical study of drag and mass transfer in creeping power law flow across tube banks, Industrial \& Engineering Chemistry Research 43 (2004) 3439-3450.

[24] A.A. Soares, J.M. Ferreira, R.P. Chhabra, Steady two-dimensional non-Newtonian flow past an array of long circular cylinders up to Reynolds number 500: numerical study, Canadian Journal of Chemical Engineering 83 (2005) 437-449.

[25] E.K. Zholkovskiy, V.N. Shilov, J.H. Masliyah, M.P. Bondarenko, Hydrodynamic cel model: general formulation and comparative analysis of different approaches, Canadian Journal of Chemical Engineering 85 (2007) 701-725.
[26] R.P. Chhabra, Bubbles, Drops and Particles in Non-Newtonian Fluids, CRC Taylor \& Francis, Boca Raton, FL, 2007.

[27] R.B. Bird, R.C. Armstrong, O. Hassager, Dynamics of Polymeric Liquids, Wiley, New York, 1977.

[28] H.A. Barnes, J.F. Hutton, K. Walters, An Introduction to Rheology, Elsevier, New York, 1989.

[29] J. Ferguson, Z. Kemblowski, Applied Fluid Rheology, Elsevier, New York, 1991.

[30] R. Clift, J. Grace, M.E. Weber, Bubbles, Drops and Particles, Academic Press, New York, 1978.

[31] R.M. Turian, An experimental investigation of flow of a aqueous non-Newtonian high polymer solutions past a sphere, AIChE Journal 13 (1967) 999-1006.

[32] R.B. Bird, W.E. Stewart, E.N. Lightfoot, Transport Phenomena, John Wiley and Sons, New York, 2002

[33] Y. Kawase, J.J. Ulbrecht, Drag and mass transfer in non-Newtonian flows through multi-particle systems at low Reynolds numbers, Chemical Engineering Science 36 (1981) 1193-1202.

[34] V. Mohan, J. Raghuraman, Bounds on the drag for creeping flow of an Ellis fluid past an assemblage of spheres, International Journal of Multiphase Flow 2 (1976) 581-589.

[35] R.P. Chhabra, J. Comiti, I. Machač, Flow of non-Newtonian fluids in fixed and fluidised beds, Chemical Engineering Science 56 (2001) 1-27.

[36] I. Machač, Z. Lecjaks, Wall effect for a sphere falling through a non-Newtonian fluid in a rectangular duct, Chemical Engineering Science 50 (1995) 143-148.

[37] P.V. Balaramakrishna, R.P. Chhabra, Sedimentation of a sphere along the axis of a long square duct filled with non-Newtonian liquids, The Canadian Journal of Chemical Engineering 70 (1992) 803-807. 